\title{
SEQUENTIAL PROCUREMENT AUCTIONS
}

\author{
Richard LUTON and R. Preston MCAFEE* \\ Department of Economics, University of Western Ontario, London, Canada N6A 5C2
}

Received June 1985, revised version received July 1986

Two auctions are held in sequence with the possibility of learning between the second and the first. The buyer optimally chooses to discriminate against the winner of the first auction in the second. The optimal mechanism has a discontinuity in the winner's second bid; this strictly dominates a sequence of independent sealed bid auctions.

\section{Introduction}

Procurement by governments accounts for 10-15 percent of national product in most Western nations [McAfee and McMillan (1985a)]. Consequently, analyses of procurement policies apply to a significant portion of economic activity. This paper considers sequential procurement: a buyer will purchase items in sequence, with costs correlated across time. Thus, if auctions are held, bids in the first auction reveal something about costs in the second. This paper, then, contributes to the optimal auction literature, with the major application in government procurement.

It is arguable that nonsequential procurement is the exception; once a government buys an item, it continues buying it for the rest of time. Governments fix roads, buy military items, plant trees, etc. every year. These all represent sequentially procured items. Often the same firms bid each year, and this is presumed here for simplicity.

Research and development projects are often followed by production projects. Generally, the winner of the R\&D project (the incumbent) has an advantage in production, for he may have learned something useful during the research that he keeps secret, or because the mere fact that he won the R\&D bidding is itself a signal of an advantage in this area. Such a situation means that the government possesses useful information: in the second project, expectations concerning the incumbent do not coincide with expect-

\footnotetext{
*The authors wish to thank, without implicating, Glenn MacDonald, Ig Horstmann, Alan Slivinski, John McMillan, attendees of the Center for Decision Sciences Theory Workshop and two anonymous referees. The Ontario Economic Council provided partial support.
} 
ations concerning rivals. This alone is sufficient to cause asymmetric, or discriminatory, treatment of incumbent and other firms.

Prior to describing the model, a few general remarks are warranted. The standard auction mode ${ }^{1}$ is the only model of competition for government contracts available, although the various principal-agent models are applicable when there is no competition. In addition, government procurement provides the best application of the optimal auction model, for it is with a government that the model's assumptions are most closely satisfied.

There are two major assumptions of the optimal auction model that are most plausible for a government. First, the agents are assumed to be risk neutral. As governments typically self-insure, and deal with large firms, this hypothesis is quite reasonable, although it is also plausible for other agents bidding for items with small value. In addition, it is presumed that the auction designer can commit to a mechanism. This means that he can bind himself to a decision rule which may not be subgame perfect, i.e. circumstances may arise where the agent wishes to renege on his commitment. Governments, by passing laws which bind themselves and are enforced by third parties (the courts), can feasibly bind themselves to mechanisms. In addition, it may be that, for a government, the repercussions of reneging are very large.

This is important because even such a simple institution as a sealed-bid auction leaves an agent with the desire to renege. In a sealed-bid auction, firms submit bids privately, and the lowest bidder is awarded the job and paid his bid. In general, it is straightforward to deduce this bidder's opportunity cost from his bid. Thus, by reneging on the commitment to pay the lowest bid, the buyer could then offer to pay the firm its cost, and the firm would agree. Of course, knowing that the buyer might do this, the firm bids higher, and no solution to the auction design problem exists.

When the buyer can commit, and the firms are risk neutral, and have identically and independently distributed costs, any of the standard auction forms minimize expected procurement costs. This includes the sealed bid auction, which is favored by all of the levels of U.S. governments. However, as we shall show, if there are two projects, and the costs in the second are reflected in the costs on the first, the independent sealed bid auctions are no longer optimal. Indeed, the optimal auction is discriminatory: it treats the incumbent and rivals differently.

The model employed is a simple learning model. There are two projects. $n$ firms have privately observed opportunity costs, drawn identically and independently from the cumulative $F$, for the first project. They bid for the

\footnotetext{
${ }^{1}$ Myerson (1981) and Riley and Samuelson (1981). See also Milgrom and Weber (1982) for an extensive analysis of these auctions in a very general setting. McAfee and McMillan (1986) provide a survey. Matthews (1983) allows for risk aversion, while Myerson (1981) discusses nonidentical (but still independent) distributions of values.
} 
project, and the lowest bid wins. Prior to the second project, each firm gets a second draw from $F$, and their costs on the second project is the minimum of the two draws. Thus, if the minimum is lower than first-period cost, the second firm has learned how to do the job more cheaply.

We derive the mechanism that minimizes the sum of expected procurement costs on the two projects. It is convenient to use the revelation principle ${ }^{2}$ and design an incentive compatible mechanism, so that payoffs are structured to provide the agent with an incentive to honestly reveal his costs.

The optimal mechanism has several interesting features. First, it is discriminatory: the winner of the first project (the incumbent) is disadvantaged in the second. In the second project, the incumbent needs significantly lower cost than his rivals in order to win. This is a policy used by Ontario Hydro [McAfee and McMillan (1985a)], Ontario's electric utility. Second, a positive fee is charged at the time of the first auction for the privilege of bidding in the second, and this extracts surplus from the rivals (that is, firms other than the incumbent), and only the incumbent has positive profits in the second period.

Third, the discrimination rule is discontinuous: a slight perturbation of the incumbent's cost causes a discrete jump in the probability of winning the contract. To understand these results, we must consider the intuition behind the standard auction. Suppose, $x_{1}, \ldots, x_{n}$ are $n$ identically and independently distributed opportunity costs with cumulative $F$, and continuous density $f$. Let $x^{1}$ and $x^{2}$ be the first and second lowest order statistics, respectively. The buyer expects to pay $x^{2}$ for the good, and buy from the firm with lowest cost (in an English auction, the buyer pays $x^{2}$ exactly, for the second-last bidder drops out at his cost $x^{2}$, so the lowest cost bidder wins the bid at price $x^{2}$ ). Furthermore, let $E$ be the expectation over the $x$ 's. Then

$$
E x^{2}=E\left(x^{1}+F\left(x^{1}\right) / f\left(x^{1}\right)\right)
$$

Thus, the function ${ }^{3}$

$$
J(x)=x+F(x) / f(x)
$$

effectively translates costs into prices paid. Suppose $x_{i}$ is the ith firm's cost draw on the first project, and $y_{i}$ the draw on the second, so that the firm's cost is

$$
z_{i}=\min \left\{x_{i}, y_{i}\right\}
$$

\footnotetext{
${ }^{2}$ Harris and Townsend (1981) and Myerson (1982).

${ }^{3}$ This corresponds to $x-[1-F(x)] /[f(x)]$ in a selling auction. See Myerson (1981), and others.
} 
Then define

$$
\psi\left(x_{i}, y_{i}\right)= \begin{cases}J\left(x_{i}\right), & x_{i} \leqq y_{i} \\ y_{i}, & y_{i}<x_{i}\end{cases}
$$

Then, on the second project, the buyer will optimally choose to buy from the firm with the smallest $\psi$. To see this, note that the buyer can charge a fee equal to the minimum second-period profits of any firm. ${ }^{4}$ The expected profits associated with winning with a $y_{i}$ can be extracted. Thus, secondperiod profits are essentially irrelevant, for they can be taxed away prior to the revelation of the $y_{i}$. However, this argument does not apply to the incumbent, simply because he may win with $x_{i}$ instead of only with $y_{i}$. Thus, his profits cannot be completely extracted (insofar as they vary with $x_{i}$, for otherwise the firm would choose to exit when expected profits were low). However, they can be reduced by discriminating against him. This is precisely what occurs. Considering $J\left(x_{i}\right)$ to be the incumbent's first-period price, a rival can beat the incumbent merely by beating his first price, instead of beating his first-period cost.

This can be seen most clearly in nonstochastic auctions. Suppose one firm was known to have lowest cost $C_{1}$. The Bertrand equilibrium is for this firm to supply the good at a price equal to the second lowest cost $C_{2}$. However, if the buyer can credibly threaten to buy from the second lowest cost firm unless a price of $C_{1}$ (plus an infinitesimal, perhaps) is offered, the low-cost firm is forced to supply at cost. This illustrates why it is useful to discriminate in favor of high-cost firms - to make low-cost firms bid more competitively.

In the stochastic setting, of course, such discrimination carries the risk of picking the higher cost supplier. In the optimal auction, this risk is balanced against the savings created by forcing low-cost firms to bid more aggressively.

Although this minimizes the buyer's procurement cost, the solution is inefficient, in that it will occasionally be the case that, if the incumbent is denoted with subscript $I$, and rivals, $R$, that

$$
x_{\mathrm{I}}<y_{\mathrm{R}}<J\left(x_{\mathrm{I}}\right)
$$

and $x_{\mathrm{I}} \leqq y_{1}$. In this instance, he picks the rival with higher cost. This is not unexpected, and can be compared with Myerson (1981). Myerson, in a oneshot auction, considers the case when $x_{i}$ is drawn from a cumulative $F_{i}$ that

\footnotetext{
${ }^{4}$ This works insofar as the firms do not know their second-period costs. If they do know their costs, as in the first period, the use of a fee is effectively the same as a reserve price. See Milgrom and Weber (1982).
} 
is not the same for each $x_{i}$. In this case, the buyer effectively chooses the firm whose $i$ minimizes $x_{i}+F_{i}\left(x_{i}\right) / F_{i}^{\prime}\left(x_{i}\right)$. By our analogy that $J$ measures prices, the Myerson auction, quite naturally, picks the firm with the lowest price. In our auction, since second-period rents are extractable insofar as they do not vary with first-period information, choosing the minimum $\psi$ chooses the lowest effective price.

One way of explaining these results involves reserve prices. In the second auction the buyer uses a reserve price $J\left(x_{1}\right)$, so that, to win, firms must beat the going price $J\left(x_{1}\right)$. Otherwise, the incumbent is selected. This reserve price is endogenous in that it arises through the first-period auction.

This paper is related to work by Palfrey (1983) on bundling of goods. Palfrey examines the decision to sell several distinct goods in a single auction, or to auction them off separately. He finds the revenue maximizing seller will choose to bundle (auction together) when there are few bidders. The analysis is executed in sealed-bid and second-price auctions and is, in general, not the optimal auction. However, the work is closely related to ours, concerning several goods at a single point in time, where ours is a single good at several points in time. In both cases the auction designer chooses to 'tie-in', or connect, the outcomes of the auctions.

Uhlike Palfrey's results, it is significant that the optimal strategy is independent of the number of bidders. That is to say, the choice of whether to choose the incumbent over rival in the second period does not depend on the number of rivals. Obviously, the extent of competition affects the probability the incumbent is selected. But the discrimination rule itself does not vary, for basically the same reason that optimal reserve prices are invariant to the number of bidders.

This intuition is quite straightforwatd, but the problem's solution is less so. Consequently, many of the paper's developments are placed in the appendix, with only the highlights included in the text.

\section{The model}

The buyer desires to have two projects done in sequence. There are $n$ firms capable of doing both projects, and the buyers and firms are assumed to be risk neutral. It is presumed that the buyer can commit to a mechanism: that is, the buyer can guarantee he will use a given decision rule, even when this rule is not, in some realization, as good for the buyer as reneging.

Each firm $i$ gets an independent draw $x_{i}$, that is private information, from a twice continuously differentiable cumulative distribution function $F, f=F^{\prime}$, satisfying

$$
\frac{\mathrm{d}}{\mathrm{d} x} x+\frac{F(x)}{f(x)} \geqq 0 .
$$


This is the usual assumption on the inverse hazard rate, for the case of buying instead of selling [Myerson (1981), Riley and Samuelson (1981)].

The buyer is presumed to use an incentive compatible mechanism [see Myerson (1982)] in order to contract the two projects. In an incentive compatible mechanism, there is a Nash equilibrium in which the agents respond honestly with their type $x_{i}$. This is without loss of optimality, by the revelation principle.

$x_{i}$ is the firm's cost of doing the first project. The first stage of the mechanism involves the buyer requesting the agent's costs, and based on the vector of reported costs, choosing an agent to do the project and paying the agents. Risk neutrality simplifies this, because the agents may be paid based on their own report $\hat{x}_{i}$, since the agent is indifferent between a risky payment (based on the other agents' reports) and its expected value. Let $q\left(\hat{x}_{i}\right)$ be the payment to agent $i$, based on the report $\hat{x}_{i}$. A hat will generally be used to distinguish a report from a true value. The agent with the smallest $\hat{x}_{i}$ is chosen to execute the project, and is thereafter called the incumbent; all other agents are called rivals. Subscripts $I$ and $R$ are used to refer to incumbent and rivals, respectively.

Prior to the initiation of project 2 , the agents obtain a second independent draw $y_{i}$ from $F$, and their second-period costs are the minimum of their two draws:

$$
z_{i}=\min \left\{x_{i}, y_{i}\right\}, \quad i=1, \ldots, n .
$$

As before, $y_{i}$ is private information. This second draw is like learning, with recall, although the learning is exogenous to the agent. The revelation principle guarantees that the buyer need not tell any firm the other firms' reports. This has a straightforward intuition. Effectively, the buyer can commit to pay the firms and choose one in such a way to mimic the firms' behaviors (in this case, their second-period reports) as if they were informed. In other words, the buyer commits to simulating the game where the firms are told the other firms' responses in a game where the firms are not told. Thus, with commitment, not telling the agents anything is without loss of generality. However, since one agent must actually do the work, that agent must know he was selected, while the others know they were not. As a result, the incumbent and rivals have different posteriors of each other's $x_{i}$ 's, since the incumbent knows his $\hat{x}_{I}$ was lowest, while the rivals know at least one agent had $x_{i}<x_{\mathrm{R}}$.

This is captured by using the incumbent's expectation operator

$$
E_{1}(\cdot)=\int_{\hat{x}_{1}} \cdots \int_{\hat{x}_{1}}(\cdot) \frac{f\left(x_{-1}\right) \mathrm{d} x_{-1}}{\left[1-F\left(\hat{x}_{1}\right)\right]^{n-1}}
$$


and the rival's expectation operator:

$$
E_{\mathrm{R}}(\cdot)=(n-1) \int_{0}^{\hat{x}_{\mathrm{R}}} \int_{x_{n}}^{x_{m}} \cdots \int_{x_{n}}^{x_{m}}(\cdot) \frac{f\left(x_{-\mathbf{R}}\right) \mathrm{d} x_{-\mathbf{R}}}{1-\left[1-F\left(\hat{x}_{\mathrm{R}}\right)\right]^{n-1}},
$$

where

$$
x_{m}=\inf \{x \mid F(x)=1\}
$$

is the largest possible $x$, and

$$
\begin{aligned}
& f\left(x_{-j}\right)=\prod_{i \neq j} f\left(x_{i}\right) \\
& \mathrm{d} x_{-j}=\mathrm{d} x_{1} \mathrm{~d} x_{2} \ldots \mathrm{d} x_{j-1} \mathrm{~d} x_{j+1} \ldots \mathrm{d} x_{n}
\end{aligned}
$$

Eq. (8) expresses the fact that the incumbent knows that all $n-1$ rivals reported costs $x_{i} \geqq \hat{x}_{1}$. Eq. (9) embodies any rival's knowledge that one firm reported cost $x_{n}$ less than $\hat{x}_{\mathrm{R}}$, and all other firms reported cost in excess of $x_{n}$.

The mechanism for the second project involves the firms reporting their new draw $y_{i}$, and then being paid $p_{i}\left(y_{i}, \bar{x}\right)$ based only on the report $y_{i}$ and the first period reports $\bar{x}$. The agent is assigned the second project with probability $\mu_{i}\left(y_{i}, x_{i}, y_{-i}, x_{-i}\right)$.

As is usual with sequential problems, we solve the second project first and then solve the first, as decisions made in the first period are exogenous in the second.

We presume that, if $y_{i}>x_{i}$, then

$$
\mu_{i}\left(y_{i}, x_{i}, y_{-i}, x_{-i}\right)=\mu_{i}\left(x_{i}, x_{i}, y_{-i}, x_{-i}\right)
$$

That is to say, if the second-period cost is irrelevantly high $\left(y_{i}>x_{i}\right)$, this does not decrease the probability of winning. This will be true in the constructed solution.

\section{The second project}

For any agent $j$, let $E_{j}$ be the expectation over $x_{-j}$ and $y_{-j}, j \in\{\mathbf{R}, \mathrm{I}\}$, and

$$
v_{j}(\hat{y}, x)=E_{j} \mu\left(\hat{y}, x, y_{-j}, x_{-j}\right)
$$

If the agent was honest in the first period, and has observed $y$ in the second, 
he obtains profits:

$$
\pi= \begin{cases}p(\hat{y})-y v(\hat{y}, x), & y \leqq x, \\ p(\hat{y})-x v(\hat{y}, x), & y>x,\end{cases}
$$

since $v$ is the probability the agent is chosen and spends $\min \{x, y\}$. Remember that a hat indicates a report, rather than the true value. The subscript $j$ is suppressed for clarity, along with $\bar{x}$ in $p$.

Incentive compatibility forces

by (13).

$$
p^{\prime}(y)= \begin{cases}y \frac{\partial v}{\partial y}(y, x), & y \leqq x \\ x \frac{\partial v}{\partial y}(y, x)=0, & y>x\end{cases}
$$

The second-order condition $\left[\right.$ take $\partial^{2} \pi /(\partial \hat{y})^{2}$ from (15), substituting (16) at $y=\hat{y}]$ is:

$$
v \text { nonincreasing in } y \text {. }
$$

Integrating (16) from $y$ to $x$ :

$$
\begin{aligned}
p(y) & =p(x)+\int_{x_{j}}^{y} s \frac{\partial v(s, x)}{\partial y} \mathrm{~d} s \\
& =p(x)-x v(x, x)+y v(y, x)+\int_{y}^{x} v(s, x) \mathrm{d} s .
\end{aligned}
$$

Eq. (13) insures this holds for $y>x$ as well. Define

$$
r(x)=p(x)-x v(y, x)+\int_{y}^{x} v(s, x) \mathrm{d} s
$$

Now suppose the agent has reported $\dot{x}$ in the first period. This is necessary since, to establish incentive compatibility holds in the first period, we must consider the effects of lying in the first period, including the effects on the second. This agent anticipates second-period profits of

$$
\begin{aligned}
\pi & =p(\hat{y})-z v(\hat{y}, \hat{x}) \\
& =r(\hat{x})+(\hat{y}-z) v(\hat{y}, \hat{x})+\int_{y}^{\hat{x}} v(s, \hat{x}) \mathrm{d} s,
\end{aligned}
$$


where

$$
\begin{aligned}
& z=\min \{x, y\}, \\
& \frac{\partial \pi}{\partial \hat{y}}=(\hat{y}-z) \frac{\partial v}{\partial \hat{y}}(\hat{y}, \hat{x}) .
\end{aligned}
$$

Thus, $\pi$ is maximized at $\hat{y}=z$, by (17), yielding profit:

$$
\pi=r(\hat{x})+\int_{z}^{\hat{x}} v(s, \hat{x}) \mathrm{d} s
$$

The firm does not know his realization of $y$ when $\hat{x}$ is chosen, and thus we need only concern ourselves with the expectation of $\pi$ over $y$. Call this $\pi^{2}(x, \hat{x})$.

\section{Lemma 1 .}

$$
\frac{\partial}{\partial x} E_{j} \pi^{2}(\hat{x}, x)=-[1-F(x)] v_{j}(x, \hat{x}), \quad j \in\{\mathbf{I}, \mathbf{R}\}
$$

The proof involves integrating (23) over $y$, and then differentiating with respect to $x$, and is provided in the appendix.

Eq. (24) is the only use of the second project in the first, but a couple of points bear mention. There is an asymmetry in the second project between the incumbent and the rivals, and the buyer will exploit this to lower secondperiod costs. The profit function is defined entirely in terms of the probability $\mu$ in expectation. Thus, second-period profits can be expressed in terms the choice variable $\mu$. We will now show that the buyer's total procurement costs can similarly be expressed. This makes the optimization problem quite straightforward.

\section{The first project}

Now consider a firm who knows his own cost $x$, and is considering a report $\hat{x}$. Given that the other agents respond honestly, his probability of becoming the incumbent is $[1-F(\hat{x})]^{n-1}$. Since $q(\hat{x})$ is the payment made, he anticipates profits of

$$
\begin{aligned}
\pi^{1}(\hat{x}, x)= & q(\hat{x})-x(1-F(\hat{x}))^{n-1}+[1-F(\hat{x})]^{n-1} E_{\mathrm{l}} \pi_{\mathrm{l}}^{2}(\hat{x}, x) \\
& +\left[1-[1-F(\hat{x})]^{n-1}\right] E_{\mathrm{R}} \pi_{\mathrm{R}}^{2}(\hat{x}, x),
\end{aligned}
$$


which is, simply, payment, minus cost of doing the first project, plus expected second-period profit.

Since the firm chooses $\hat{x}$ to maximize $\pi^{1}(\hat{x}, x)$, the envelope theorem yields:

$$
\begin{aligned}
\frac{\mathrm{d}}{\mathrm{d} x} \pi^{1}(x, x)= & \left.\frac{\partial}{\partial x} \pi^{1}(\hat{x}, x)\right|_{\hat{x}=x} \\
= & -\left[(1-F(x))^{n-1}+[1-F(x)]^{n} v_{\mathbf{I}}(x, x)\right. \\
& \left.+\left[1-(1-F(x))^{n-1}\right](1-F(x)) v_{\mathbf{R}}(x, x)\right] .
\end{aligned}
$$

By integrating (26) and shifting integrals, using (8), (9), and (14), we obtain that expected profits $n E \pi^{1}$, satisfies

\section{Theorem 1}

$$
\begin{aligned}
n E \pi^{1}= & n \int_{0}^{x_{m}} F(x)[1-F(x)]^{n-1} \mathrm{~d} x \\
& +\sum_{i=1}^{n} \int_{0}^{x_{m}} \cdots \int_{0}^{x_{m}} \mu_{i}\left(x_{i}, x_{i}, y_{-i}, x_{-i}\right) \\
& \times \frac{F\left(x_{i}\right)\left(1-F\left(x_{i}\right)\right)}{f\left(x_{i}\right)} f(\bar{x}) f\left(y_{-i}\right) \mathrm{d} \bar{x} \mathrm{~d} y_{-i} .
\end{aligned}
$$

Furthermore, the buyer's expected payment is

$$
\begin{aligned}
\Phi= & \int_{0}^{x_{m}}\left[x+\frac{F(x)}{f(x)}\right] n[1-F(x)]^{n-1} f(x) \mathrm{d} x \\
& +\sum_{i=1}^{n} \int_{0}^{x_{m}} \cdots \int_{0}^{x_{m}} \psi\left(x_{i}, y_{i}\right) \mu_{i}\left(x_{i}, y_{i}, x_{-i}, y_{-i}\right) f(\bar{x}) f(\bar{y}) \mathrm{d} \bar{x} \mathrm{~d} \bar{y}
\end{aligned}
$$

where

$$
\psi\left(x_{i}, y_{i}\right)=\left\{\begin{array}{cl}
y_{i}, & \text { if } y_{i}<x_{i}, \\
x_{i}+\frac{F\left(x_{i}\right)}{f\left(x_{i}\right)}, & \text { if } y_{i} \geqq x_{i} .
\end{array}\right.
$$

The proof is tedious, and left for the appendix.

Recall the intuition given that $\psi$ is the effective price the principal is paying, when agents have costs $x_{i}$ and $y_{i}$. Eq. (28) expresses this directly, for 
the second term is precisely the expected value of $\psi$, given $\mu$ is the probability of choosing the ith firm, and $f(\bar{x}) f(\bar{y})$ is the density of the $\left(x_{i}, y_{i}\right), i=1, \ldots, n$. The first term is the second-order statistic, and thus the expected payment on the first project.

One way of understanding this is that a reserve price $J\left(x_{\mathrm{I}}\right)$ is operated in the second auction, and the lowest $y_{i}$ wins if it is less than the reserve price, and otherwise the incumbent is selected. In addition, the rescrve pricc is endogenous, depending on the first auction's outcome. To make this incentive compatible, of course, the payment function $p\left(y_{i}, \bar{x}\right)$ is discontinuous as well, since the profit function is continuous.

The buyer picks the lowest $\psi$ because $\mu_{i}$ is a probability, and therefore non-negative and constrained by

$$
\sum_{i=1}^{n} \mu_{i}=1
$$

Minımizing (29) subject to (30) is immediate.

The proof of the theorem does contain one technical development of note. By the use of the envelope theorem on profit, it is quite simple to express profit as an expectation over $\mu$ and the densities. Then, with some notationally complicated but straightforward rearrangement of limits of integration, one obtains expression (29).

\section{Conclusion}

There are several major conclusions to be drawn from this model, that should generalize to more complex linked sequences of projects. First, a fee to bid on the second project will be charged at the time of the first project, and will generally be decreasing in costs reported on the first project [this follows from (25)]. It will not succeed in extracting all the rents, for the winner of the first project will generally acquire second-period rents.

Second, the buyer will use a discrimination rule in the second period that is unfavorable to the incumbent: the incumbent needs lower cost to win than a rival. Indeed, the rival need only beat the incumbent's first-period price to win, while the incumbent must beat his own first-period cost.

The discrimination rule is not continuous, and thus the payment function is not, as well, in the second period. This is precisely the rule by Ontario Hydro in practice, for they require the incumbent to lower his cost by 15 percent in order to continue [see McAfee and McMillan (1985a) for a wealth of institutional details].

It is useful to conceal the bids made in the first auction from the other bidders. The reason is that, otherwise, the firms will know $x_{1}$, and the secondperiod payments will depend directly on $x_{i}$. Without revealing this inform- 
ation, only the incumbent's payment will depend on $x_{1}$. Of course, the optimal mechanism can be implemented with revelation of $x_{1}$. However, second-period payments to rivals will, in general, depend on $x_{1}$, being given by $(18)$.

There is a stylized fact that we see the same firms winning contracts from the government because of some official malfeasance, bribery or the like. This model suggests a different explanation: winning the first contract of a sequence is a signal of comparative advantage, and so one expects to see the firm win more often in future contracts.

If the inefficiency of the optimal auction is an objection, it should be noted that the normal sealed bid auction is, itself, inefficient. The reason is, since the incumbent and rival have different conditional probabilities of winning, they will bid differently. Indeed, at $\operatorname{cost} z_{1}=x_{1}$, the incumbent expects positive profits, since he has a positive probability of winning. A rival, however, has a zero probability of winning at this cost, and thus expects zero profits at a bid of $x_{1}$ (this is true when $x_{1}$ is announced. If $x_{1}$ is not announced, then this will change the outcome). Thus, it follows that the firms bid differently in a sealed bid auction. For a more intensive analysis of discrimination in sealed bid auctions, see McAfee and McMillan (1985b).

Finally, in some realizations, the incumbent's first-period cost may be completely ignored. This occurs whenever $J\left(x_{1}\right) \geqq x_{m}$. In such a case, the buyer provides incentives so that the incumbent competes on an equal basis with the rivals, and his former advantage ignored.

\section{Appendix}

Proof of Lemma 1

$$
\begin{aligned}
\pi^{2}(\hat{x}, x)= & \int_{0}^{x_{m}} \pi f(y) \mathrm{d} y \\
= & r(\hat{x})+\int_{0}^{x_{m}} \int_{m i n}^{\hat{x}} v(x, y\} \\
= & r(\hat{x})+\int_{0}^{x} \int_{y}^{\hat{x}} v(s, \hat{x}) \mathrm{d} s f(y) \mathrm{d} y \\
= & r(\hat{x})+\left.F(y) \int_{y}^{\hat{x}} v(s, \hat{x}) \mathrm{d} s\right|_{0} ^{x}+\int_{0}^{x} F(y) v(y, \hat{x}) \mathrm{d} y+\int_{x}^{x_{m}} \int_{x}^{\hat{x}} v(s, \hat{x}) \mathrm{d} s f(y) \mathrm{d} y \\
& +[1-F(x)] \int_{x}^{\hat{x}} v(s, \hat{x}) \mathrm{d} s \\
= & r(\hat{x})+\int_{x}^{\hat{x}} v(s, \hat{x}) \mathrm{d} s+\int_{0}^{x} F(y) v(y, \hat{x}) \mathrm{d} y .
\end{aligned}
$$


Thus,

$$
\frac{\partial \pi_{j}^{2}(\hat{x}, x)}{\partial x}=-(1-F(x)) v_{j}(x, \hat{x})
$$

Proof of the theorem. Note that $\pi^{1}\left(x_{m}, x_{m}\right)$ can be set to zero, since $\pi^{1}(x, x)$ is nonincreasing by (26), and, were $\pi^{1}\left(x_{m}, x_{m}\right)>0$, a uniform fee can be charged equal to $\pi^{1}\left(x_{m}, x_{m}\right)$, for the right to participate. All firms would be willing to pay this fee. Thus, expected profit is

$$
\begin{aligned}
& E \pi^{1}=\int_{0}^{x_{m}} \pi^{1}(x, x) f(x) \mathrm{d} x \\
& =\left.F(x) \pi^{1}(x, x)\right|_{0} ^{x_{m}}-\int_{0}^{x_{m}} F(x)\left[\frac{\mathrm{d}}{\mathrm{d} x} \pi^{1}(x, x)\right] \mathrm{d} x \\
& =\int_{0}^{x_{m}} F(x)[1-F(x)]^{n-1} \mathrm{~d} x \\
& +\int_{0}^{x_{m}} F(x)(1-F(x))\left[[1-F(x)]^{n-1}\right. \\
& \left.\left.\times E_{\mathrm{l}} v_{1}(x, x)+\left[1-(1-F(x))^{n-1}\right] E_{1} v_{\mathrm{R}}(x, x)\right]\right] \mathrm{d} x \\
& =\int_{0}^{x_{m}} F(x)[1-F(x)]^{n-1} \mathrm{~d} x \\
& +\int_{0}^{x_{m}} F(x)(1-F(x)) \int_{x}^{x_{m}} \cdots \int_{x}^{x_{m}} \int_{0}^{x_{m}} \cdots \int_{0}^{x_{m}} \\
& \times \mu_{\mathrm{I}}\left(x, x, y_{-i}, x_{-i}\right) f\left(y_{-i}\right) f\left(x_{-i}\right) \mathrm{d} y_{-i} \mathrm{~d} x_{-i} \mathrm{~d} x \\
& +(n-1) \int_{0}^{x_{m}} F(x)(1-F(x)) \int_{0}^{x} \int_{x_{n}}^{x_{m}} \cdots \int_{x_{n}}^{x_{m}} \int_{0}^{x_{m}} \cdots \int_{0}^{x_{m}} \\
& \times \mu_{\mathrm{R}}\left(x, x, y_{-i}, x_{-i}\right) f\left(y_{-i}\right) f\left(x_{-i}\right) \mathrm{d} y_{-i} \mathrm{~d} x_{-i} \mathrm{~d} x \\
& =\int_{0}^{x_{m}} F(x)[1-F(x)]^{n-1} \mathrm{~d} x \\
& +\int_{0}^{x_{m}} \int_{x_{n}}^{x_{m}} \cdots \int_{x_{n}}^{x_{m}} \int_{0}^{x_{m}} \cdots \int_{0}^{x_{m}} C_{x_{n} \leqq y_{n}} \frac{F\left(x_{n}\right)}{f\left(x_{n}\right)}
\end{aligned}
$$




$$
\begin{aligned}
& \times \mu_{\mathrm{I}}\left(y_{n}, x_{n}, y_{-n}, x_{-n}\right) f(\bar{y}) f(\bar{x}) \mathrm{d} \bar{y} \mathrm{~d} \bar{x} \\
& +(n-1) \int_{0}^{x_{m}} \int_{x_{n}}^{x_{m}} \cdots \int_{x_{n}}^{x_{m}} \int_{0}^{x_{n}} \cdots \int_{0}^{x_{m}} C_{x_{i} \leqq y_{i}} \frac{F\left(x_{i}\right)}{f\left(x_{i}\right)} \\
& \times \mu_{\mathrm{R}}\left(y_{i}, x_{i}, y_{-i}, x_{-i}\right) f(\bar{y}) f(\bar{x}) \mathrm{d} \bar{y} \mathrm{~d} \bar{x} \\
& =\int_{0}^{x_{m}} F(x)[1-F(x)]^{n-1} \mathrm{~d} x \\
& +\sum_{i=1}^{n} \int_{0}^{x_{m}} \int_{x_{n}}^{x_{m}} \ldots \int_{x_{n}}^{x_{m}} \int_{0}^{x_{m}} \cdots \int_{0}^{x_{n}} C_{x_{i} \leqq y_{i}} \frac{F\left(x_{i}\right)}{f\left(x_{i}\right)} \\
& \times \mu_{i}\left(y_{i}, x_{i}, y y_{-i}, x x_{-i}\right) f(\bar{y}) f(\bar{x}) \mathrm{d} \bar{y} \mathrm{~d} \bar{x},
\end{aligned}
$$

where

$$
C_{x \leqq y}= \begin{cases}0, & x \geqq y, \\ 1, & x \leqq y,\end{cases}
$$

using (13), and Fubini's Theorem.

Note, as well, that

$$
\begin{aligned}
n E \pi^{1}= & n \int_{0}^{x_{m}} F(x)[1-F(x)]^{n-1} \mathrm{~d} x \\
& +\sum_{i=1}^{n} \int_{0}^{x_{m}} \cdots \int_{0}^{x_{m}} C_{x_{i} \leqq y_{i}} \mu_{i}(\bar{y}, \bar{x}) \frac{F\left(x_{i}\right)}{f\left(x_{i}\right)} f(\bar{y}) f(\bar{x}) \mathrm{d} \bar{y} \mathrm{~d} \bar{x},
\end{aligned}
$$

since the previous expression differs from this only by the construction that $x_{n}$ was the lowest of the $x_{i}^{\prime}$ 's (probability $1 / n$ ).

The total cost incurred is, in the second period:

$$
\sum_{i=1}^{n} \int_{0}^{x_{m}} \cdots \int_{0}^{x_{m}} z_{i} \mu_{i}(\bar{y}, \bar{x}) f(\bar{x}) f(\bar{y}) \mathrm{d} \bar{x} \mathrm{~d} \bar{y}
$$

since $z_{i}$ is the cost incurred by the $i$ th agent, and this happens with probability $\mu_{i}(\bar{y}, \bar{x})$. Therefore, since expected payment $\Phi$ must be expected cost plus expected profit:

$$
\Phi=\sum_{i=1}^{n} \int_{0}^{x_{m}} \cdots \int_{0}^{x_{m}}\left[z_{i}+C_{x_{i} \leqq y_{i}} \frac{F\left(x_{i}\right)}{f\left(x_{i}\right)}\right] f(\bar{x}) f(\bar{y}) \mathrm{d} \bar{x} \mathrm{~d} \bar{y} \text {. Q.E.D. }
$$




\section{References}

Harris, Milton and Robert Townsend, M., 1981, Resource allocation under asymmetric information, Econometrica 49, 33-64.

Matthews, Steven A., 1983, Selling to risk averse buyers with unobservable tastes, Journal of Economic Theory 30, 370-400.

McAfee, R. Preston and John McMillan, 1985a, Incentives in government contracting, University of Toronto Press.

McAfee, R. Preston and John McMillan, 1985b, Discrimination in auctions, Discussion paper no. 85-7, (University of California, San Diego), Jan.

McAfee, R. Preston and John McMillan, 1986, Auctions and bidding, Journal of Economic Literature, forthcoming.

Milgrom, Paul R, and Robert J. Weber, 1982, A theory of auctions and competitive bidding, Econometrica 50, 1089-1122.

Myerson, Roger B., 1981, Optimal auction design, Mathematics of Operations Research 6, 58-73.

Myerson, Roger B., 1982, Optimal coordination mechanisms in generalized principal-agent problems, Journal of Mathematical Economics 10, 67-81.

Palfrey, Thomas R., 1983, Bundling decisions by a multiproduct monopolist with incomplete information, Econometrica 51, 463-484.

Riley, John G. and William F. Samuelson, 1981, Optimal auctions, American Economic Review $71,381-392$ 